\title{
MARSCat: Imaging of the Martian Ionosphere using a CubeSat Constellation
}

\author{
Edgar A. Bering ${ }^{*}$, Laila Andersson ${ }^{\dagger}$, Mark Moldwin, Paul Withers ${ }^{\S}$,
}

\begin{abstract}
The MarsCAT (Mars Array of ionospheric Research Satellites forming the Constellation for Aeronomy and Tomography) Mission is a multi 6U CubeSat mission to study the ionosphere of Mars for a future mission of opportunity. The mission will investigate the plasma and magnetic structure of the Martian ionosphere, including transient plasma structures, magnetic field structure and dynamics, and energetic particle activity. The transit plan calls for a piggy back ride on a major mission using a solar electric propulsion burn for Mars Orbit Insertion (MOI). MarsCAT will make correlated multipoint studies of the ionosphere and magnetic field of Mars. Specifically, the several spacecraft will make in situ observations of the plasma density, temperature, and convection in the ionosphere of Mars. Multipoint observations are crucial requirements for attaining all the major science goals. They will also make total electron content measurements along the line of sight between the various spacecraft and simultaneous 3-axis local magnetic field measurements in several locations. The MarsCAT CubeSats will have five science instruments: a 3-axis DC magnetometer, a double-Langmuir probe, a Thermal Ion Analyzer, a solid state energetic particle detector, and interspacecraft total electron content radio occulation experiment. The MarsCAT spacecraft will be solar powered and equipped with a plasma thruster that can provide up to $4.8 \mathrm{~km} / \mathrm{s}$ of $\Delta v$, which is sufficient to achieve Mars orbit using a major mission piggyback. They have an active attitude control system, using a sun sensor and flight-proven star tracker for attitude determination, and momentum wheels for 3-axis attitude control.
\end{abstract}

\section{IV.Introduction}

$\mathrm{M}$ ARS has been a source of fascination and wonder for millennia. The main focus of the first 50 years of in situ Mars probes has been to understand the geological history of the surface of Mars. The latest US orbiter, MAVEN, is the first probe designed to study the Mars' ionosphere ${ }^{1}$. The MarsCAT mission described here will also study the Martian ionosphere as a cohesive multipoint mission. We will demonstrate the MarsCAT mission can address a set of exciting and important scientific questions that have been posed by the MAVEN data.

\section{Science Objectives and Rationale}

Many missions to Mars have helped to show the rich and ever-changing nature of the plasma environment around Mars. Together, the absence of a strong global-scale field and the presence of localized regions of crustal magnetization ensure that the magnetosphere and ionosphere of Mars display unique behavior not seen anywhere else in the solar system. The need to determine how the ionosphere varies on small spatial and temporal scales motivates the three major science goals of this mission concept.

- How does the ionosphere respond to solar wind dynamics?

- What small scale structures exist in the lower ionosphere of Mars?

- How is the ionosphere on the nightside maintained?

\footnotetext{
${ }^{*}$ University of Houston, 3507 Cullen Blvd.,\#617/PHYS 5005, Houston, TX, 77204, USA.

${ }^{\dagger}$ University of Colorado, LASP, 3665 Discovery Drive, Campus Box 590, Boulder, CO, 80303, USA.

${ }^{\ddagger}$ University of Michigan, Space Research Building, 2455 Hayward St Ann Arbor, MI, 48109, USA.

${ }^{\S}$ Boston University, 725 Commonwealth Ave Boston, MA, 02215, USA.
} 

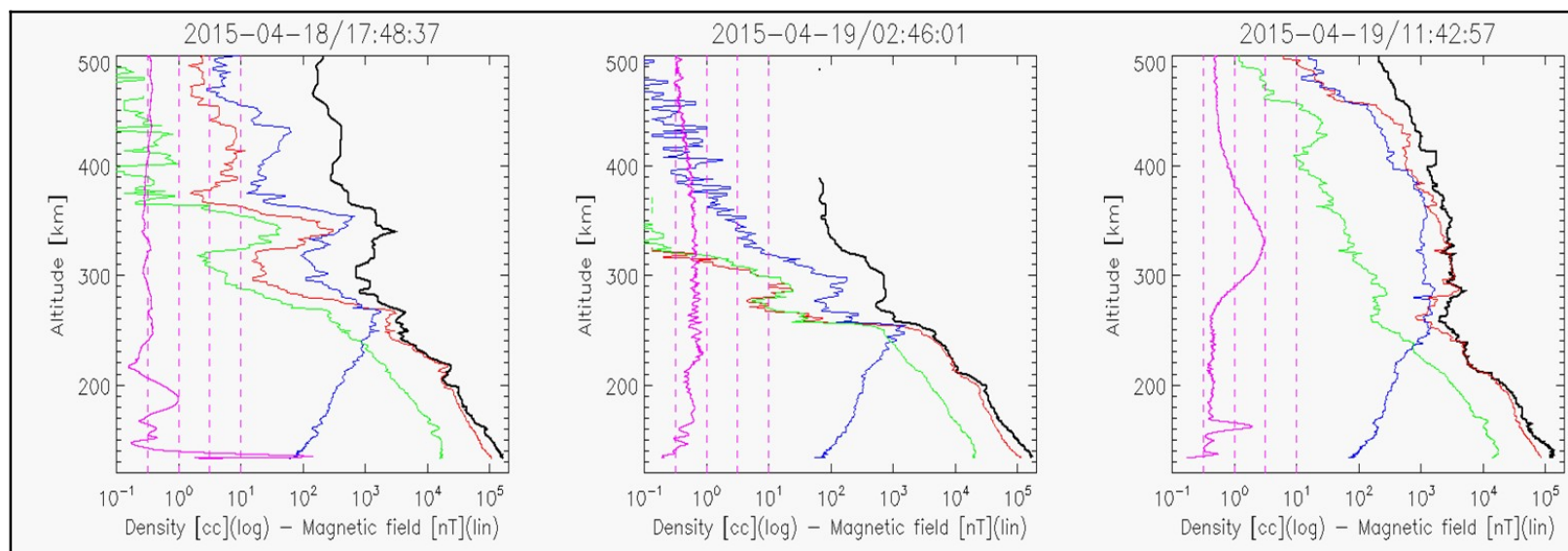

Figure 1. Density and magnetic field profiles of 3 inbound passes by the MAVEN spacecraft close to subsolar point (information presented here is only measured every other orbit). The densities are shown in logarithmic scale e-, $\mathrm{O2}+, \mathrm{O}+$ and $\mathrm{CO}+$ represented by black, red, blue, and green lines. The total magnetic field is shown in linear scale by the pink lines; where the vertical lines represent 50, 100, 150, and $200 \mathrm{nT}$. Light ion densities $\left(\mathrm{eg} \mathrm{H}^{+}\right)$are not shown and account for differences between ion and e- densities. In panel $b$, MAVEN was in the solar wind above $380 \mathrm{~km}$, as indicated by the cutoff of the $\mathrm{N}_{\mathrm{e}}$ data.

Discoveries by the MAVEN mission ${ }^{2}$ have emphasized the dynamic and complex nature of the Martian ionosphere ${ }^{3}$. Figure 1, which shows vertical profiles of electron and ion densities and magnetic field from three closely--spaced orbits from the MAVEN mission. It is clear that large changes in ionospheric conditions occur on time scales much shorter than the $\sim 5$ hour orbital period. Understanding the complex interaction of the Mars space environment with the solar wind and with the crustal field of the planet requires high cadence observations. Given the ever--increasing pace and sophistication of exploration activity at Mars, it is important to establish the extent to which the ionosphere will impact navigation and communications. That requires knowledge of how the ionosphere varies over small spatial and temporal scales that no mission has yet provided. The MarsCAT mission will acquire multipoint measurements from a constellation of CubeSats that will determine this operational risk and answer significant scientific questions about how the unique magnetic fields of Mars affect the planet's surrounding plasma environment.

\section{A. How does the ionosphere respond to solar wind dynamics?}

On the dayside, the lower ionosphere, which encompasses the main ionosphere peak and contains $>80 \%$ of the plasma, is understood. It is only above $\sim 180 \mathrm{~km}$ that plasma transport becomes a significant process. Here plasma densities are elevated above those expected from photochemical processes alone. Above $\sim 300 \mathrm{~km}$, ionospheric densities are remarkably uniform with altitude (Fig 1a). There is often no ionopause, or sharp upper boundary at which plasma densities abruptly decrease. Instead, the shocked solar wind inside the bow shock frequently compresses the dayside ionosphere significantly (Fig 1b). In this example, the shocked solar wind penetrated to 350 $\mathrm{km}$ altitude as indicated by the cutoff of the $\mathrm{N}_{\mathrm{e}}$ data and the heaviest ions $\left(\mathrm{O}_{2}+, \mathrm{CO}_{2}+\right)$ are eroded away down to altitudes as low as $300 \mathrm{~km}$. Such changes occur rapidly, as the bulk of the upper ionosphere departs and returns (Fig 1c) in less than MAVEN's five hour orbital period. The single-point measurements of MAVEN preclude robust separation of temporal and spatial effects. MarsCAT will be able to determine whether such dramatic changes in the upper ionosphere occur over the entire ionosphere on short timescales or in localized regions with potentially longer timescales. Given the complex topology of the draped solar wind magnetic field interacting with localized crustal fields ${ }^{4}$, variations on small spatial scales are a viable possibility. MarsCAT will measure local electron density at each spacecraft and also the integrated line-of-sight electron density between each spacecraft. The combination of these measurements will be very useful for identifying spatial variations in the ionosphere.

\section{B. What small-scale structures exist in the lower ionosphere of Mars?}


MAVEN and pre-MAVEN observations have shown that small-scale variations in plasma density are common in the ionosphere of Mars 5 . At Earth, such fluctuations degrade the effectiveness of radio navigation and communications systems. The corresponding effects at Mars are uncertain due to inability in current observations to distinguish spatial and temporal variations. The MarsCAT constellation of spacecraft will provide the observations needed to assess the impact of Mars plasma fluctuations on radio nav/comm systems.

The MAVEN mission has demonstrated that Rayleigh Taylor (RT) instability ${ }^{6}$ can grow at Mars. Observations below $200 \mathrm{~km}$ suggest this instability mainly occurs at dawn and dusk but potentially also at noon The dayside density profile of Figure 1a has higher density at $350 \mathrm{~km}$ than at $300 \mathrm{~km}$ suggesting if this profile is RT unstable then plasma bubbles can be created here.

\section{How is the ionosphere on the night side maintained?}

Without ionization from sunlight, densities in the nightside ionosphere might be expected to decrease rapidly ${ }^{7}$. Yet significant densities have been observed on the deep nightside. Idealized modeling suggests that constant electron precipitation associated with field-aligned currents (FAC) could maintain the observed nightside densities. However, fluxes of precipitating electrons, which produce aurora, are neither constant nor uniform. It remains unclear whether temporally and spatially varying fluxes of precipitating electrons can maintain the nightside ionosphere. FAC are often observed on the outer edges of crustal field regions where the field geometry has a boundary between closed and open regions. Density variations of a factor of 6 have been observed over a distance of $4 \mathrm{~km}$, which indicates there is small-scale structure in the ionosphere.

The night-side observations by MarsCAT will allow a better understanding of FAC and the source of the night side ionosphere. MarsCAT will be able to evaluate how important crustal fields are for FAC that can create aurora ${ }^{8}$.

\section{Mission Concept}

The mission concept is that MarsCAT will address these challenges by placing several identical CubeSats into orbit around Mars. Each CubeSat will carry a magnetometer to measure the magnetic field, a plasma analyzer to measure the thermal ion density, temperature and velocity, a Langmuir probe to measure electron density and temperature, and radios to measure electron densities between any two spacecraft.

The greatest opportunity lies in the ability to host such a mission as a secondary payload to a major mission. If possible, having the host mission provide the Mars orbit insertion (MOI) will greatly increase science options. However, each spacecraft will carry a plasma thruster that will provide autonomous capture and insertion capabilities. Each satellite will have a 3-axis attitude determination and control system capable of attitude solutions in interplanetary space and near Mars, and an embedded radio navigation system utilizing the navigation capabilities of the Mars Relay's CCSDS protocol.

The preliminary strawman orbit has periapis below $180 \mathrm{~km}$ with an inclination near $60^{\circ}$. Solar wind response studies require an eccentric orbit to be in the solar wind at apoapsis. At the same time, peak ionospheric density lies near $150 \mathrm{~km}$, the crustal fields fall off rapidly with altitude, and instability driven plasma transport has been observed by MAVEN below $200 \mathrm{~km}$ (Figure 2). Therefore, periapsis should be at or below $180 \mathrm{~km}$ for some

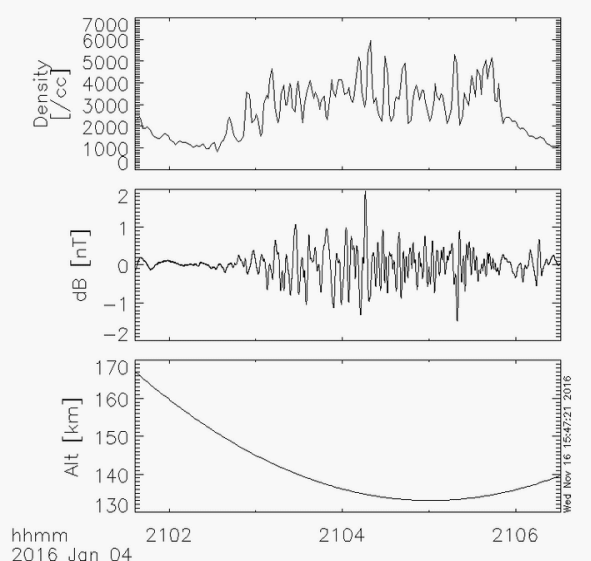

Figure 2. An example of observation of the Rayleigh-Taylor instability by MAVEN ${ }^{8}$. Panel 1 shows the perturbed density enhancements. Panel 3 is the altitude. As can be seen, MAVEN is under-sampling these phenomena. MarsCat will have the data cadence to resolve similar observations. time, in order to take data in this critical region.

There are trade-offs between the available carry mass, ability to resolve space and time variations or at least recognize them, and maximizing science return from the Total Electron Content (TEC) system. For example, variation disambiguation is easiest if one places two or more platforms in a string-of-pearls configuration, whereas the TEC system may perform better if we have two platforms flying at the same altitude in non-coplanar but otherwise identical orbits. Crustal field observations are optimized by having periapsis at $\sim 60^{\circ} \mathrm{S}$, since that is where the strongest crustal fields are located. 


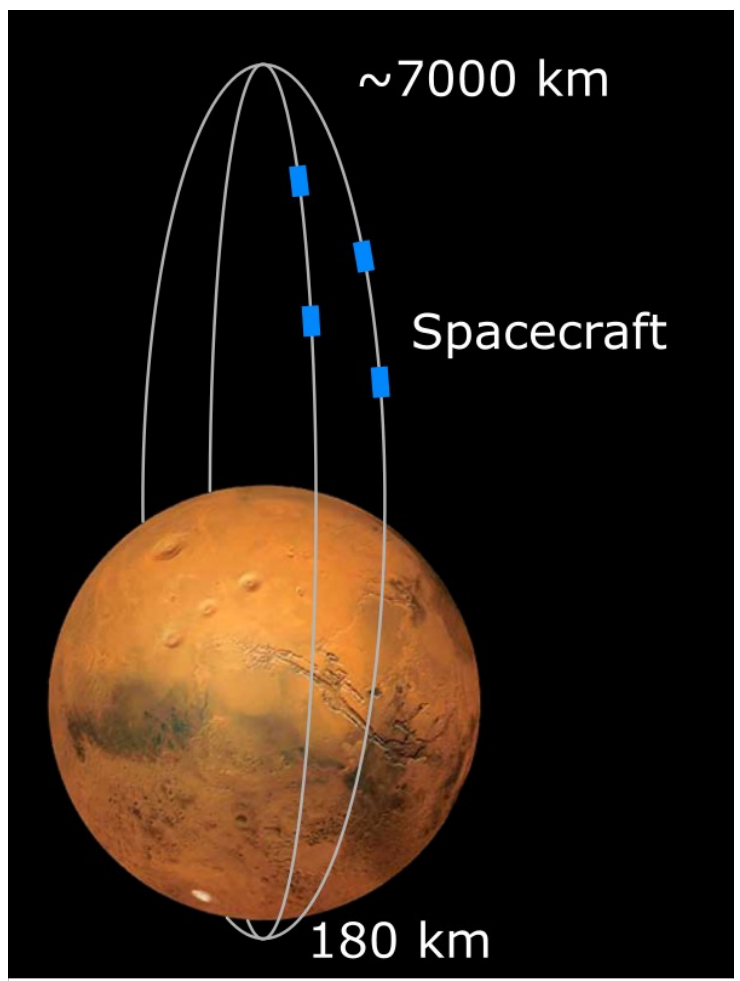

Figure 3. Orbital configuration of MarsCAT
The plasma thruster reaction mass budget may allow for up to $\sim 4 \mathrm{~km} \mathrm{~s}^{-1}$ of $\Delta v$, which enables a sequence of orbital configurations designed to study separate questions at different times during the mission. A possible configuration is shown in Figure 3. This figure shows four platforms, two each in a pair of string-of-pearls orbits that are separated by $15^{\circ}$ in Right Ascension of the Ascending Node (RAAN). The spacecraft will have elliptical orbits with periapsis at or below $180 \mathrm{~km}$ and apoapsis at $7000 \mathrm{~km}$ such that they sample the solar wind, magnetosphere, and ionosphere. Each satellite is anticipated to be an equivalent 6-U Cubesat structure.

\section{Measurements to be Taken}

The anticipated mission will place a premium on reliability in a compact and robust configuration. It will therefore utilize existing technologies wherever possible and refine those technologies when required to achieve the mission goals.

The several MarsCAT spacecraft will have identical instrumentation. They carry an induction Magnetometer (iMAGS) to measure the DC and ULF magnetic wave field, a Thermal Ion Analyzer Analyzer to measure the electron and ion density, temperature and flow velocity, double-Langmuir Probes (LP) to measure the cold electron plasma density and two Medipix radiation detectors, each with dual orthogonal sensor assemblies to measure the charged particle radiation field and characterize the X-ray bremsstrahlung from below. In addition to these instruments, MarsCAT carries a dual frequency radio package to provide total electron content information between the several spacecraft.

\section{Mission Implementation}

\section{A. Concept of Operations}

MarsCAT consists of several identical 6U CubeSat spacecraft. The best option for the MarsCAT spacecraft is to launch piggyback on a major mission and remain stowed onboard until reaching Mars. They will jettison from the mission carrier at the edge of Mars' gravitational sphere of influence and use plasma thrusters to achieve capture and Mars orbital insertion (MOI). First, even with a plasma thruster propulsion system, the amount of propellant required for independent transit to Mars by each spacecraft, followed by MOI to a relevant science orbit, would be prohibitive given the constraints of a $6 \mathrm{U}$ form factor. Second, it was deemed that the risk of both spacecraft navigating a transit trajectory to Mars independently and reliably arriving within a reasonable time window for multipoint science observations was too high. Third, mitigating the risk of additional radiation exposure on an independent transit (without the shielding mass of a pod carrier) was infeasible within the available $6 \mathrm{U}$ mass constraint. For these reasons, MarsCAT proposes to launch with a major mission.

Each MarsCAT spacecraft will be equipped with a plasma thruster like the PhaseFour RFT Thruster (RFT), a 3axis attitude determination and control system capable of attitude solutions in interplanetary space and near Mars, and an embedded radio navigation system utilizing the navigation capabilities of the Mars Relay's CCSDS protocol. Upon ejection from the carrier spacecraft at Mars' gravitational sphere of influence (500,000 km altitude), the RFT performs a finite continuous maneuver to transition from MOI to a highly elliptical, stable orbit of $200 \mathrm{x} 7000 \mathrm{~km}$ and $60^{\circ}+/ 5^{\circ}$ inclination. The MarsCAT orbits will lie in two planes, separated by approximately $15^{\circ}$ in right ascension of the ascending node (RAAN).

\section{B. Science Instruments Overview}

The structure and dynamics of the plasma and magnetic field environment around Mars is determined by interaction of solar wind and the Martian ionosphere and crustal magnetic field. Therefore, four instruments are needed to determine the local solar wind and Mars ionospheric properties and to address all of the science requirements: a magnetometer, two plasma experiments, and a remote sensing radio experiment. The instruments share a common spacecraft bus interface, spacecraft DPU, and ground system operations. 
IV. Magnetometer

The Induction Magnetometer (iMAG) to be flown on the several MarsCAT spacecraft is a new magnetometer design. Important assets of the iMAG magnetometer are its inherent linearity (with nonlinearities significantly smaller

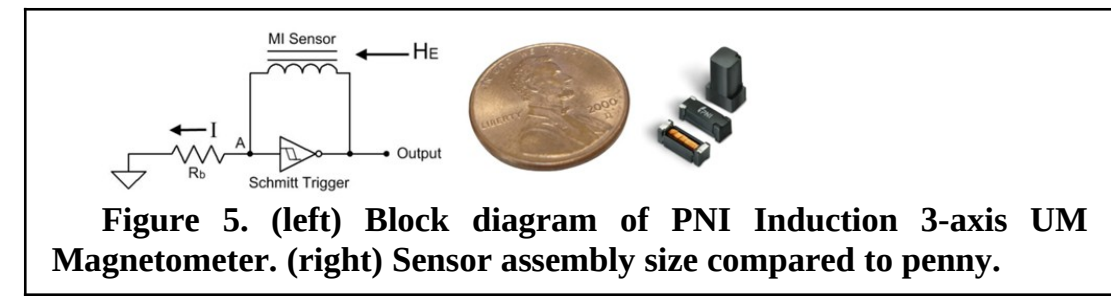

than 1 part in $10^{4}$ ) and the reliability and stability of the gains and offsets. This instrument, which is a completely different design from the traditional fluxgate magnetometer design, is inherently rad hard, low mass, low power and temperature insensitive. The electronics functional block diagram is shown in Figure 5. There is no microprocessor in this simple design.

The current circuit design realizes significant reductions in power, mass and size. Additional mass savings have also been achieved by the use of extremely small sensors and the elimination of the boom and sensor boom cable. Figure 5 shows an engineering version of the iMAG sensor. As seen in the functional diagram in Figure 5 left, the magnetometer is a LR circuit with a Schmitt Trigger for counting pulses. In Figure 5, $\mathrm{H}_{\mathrm{E}}$ is the external magnetic field parallel to the coil. The total field that the sensor experiences is due to the external field and the field generated by the circuit $\left(\mathrm{H}=\mathrm{kI}+\mathrm{H}_{\mathrm{E}}\right.$, where $\mathrm{k}$ is a property of the sensor, and $\mathrm{I}$ is the current through the circuit). The Schmitt trigger causes the current through the circuit to oscillate as the voltage bases a set "trigger" value. The time between oscillations or trigger flips is dependent on the strength of the external field and therefore the DC field can be measured by simply counting the number of flips or triggers. In fact the entire three-axis magnetometer can be implemented on one $1 \mathrm{~cm}$ x $2 \mathrm{~cm}$ printed circuit board. 


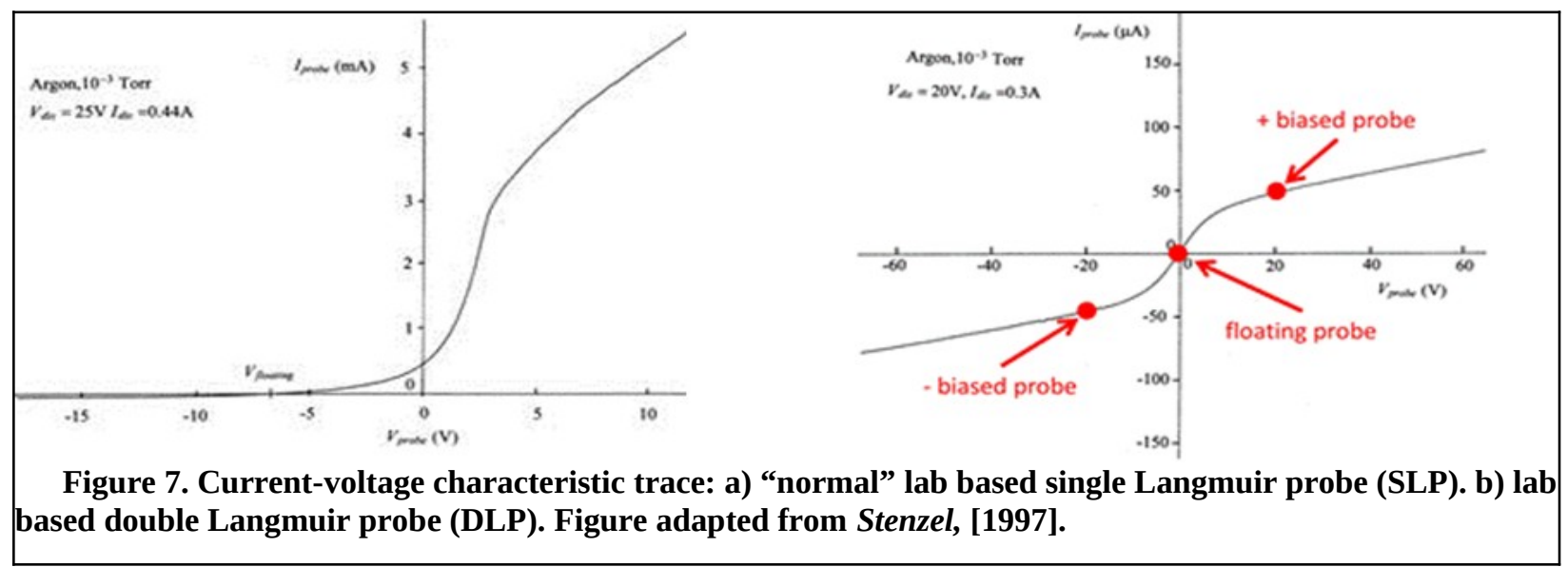

One hidden cost of fluxgate magnetometers is the need for a boom. By driving down the resource needs and cost of the magnetometer, a new approach can be incorporated in MarsCAT that eliminates the need for a boom ${ }^{9}$. This approach places several magnetometers inside and on the bus to be able to identify spacecraft magnetic signals in the data so that the external field can be recovered with processing and careful magnetic cleanliness and characterization prior to launch.

\section{Plasma Thermal Ion Analyser}

An instrument to measure the major thermal ion constituent densities, temperature and velocity is patterned after successful such sensors that have flown in many earth orbiting missions. Of greatest relevance to the proposed concept is a dual function ion velocity meter (IVM) that measures both the ion temperature and the bulk ion drift vector and occupies a small volumetric envelope compatible with a CubeSat. Figure 6 shows an isometric view of the sensor, which has an unrestricted view approximately along the satellite velocity vector.

It utilizes a square entrance aperture and a segmented collector, to measure the ion flux on each segment as a

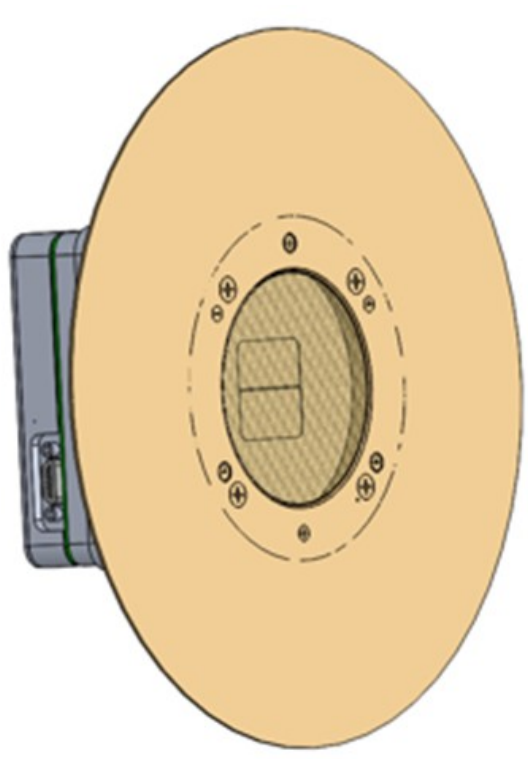

Figure 6. Thermal ion analyzer function of a retarding potential that is applied internally to a planar grid to control the energy of the ions that have access to the collector. The sensor aperture and internal electronics are electrically isolated from the spacecraft power system and the floating aperture potential provides an independent ground reference for the sensor potentials that is close to the plasma potential and independent of the spacecraft ground. In Mars orbit the ions flow supersonically into the sensor and the collector current measured as a function of retarding voltage may be fit to extract the ion temperature and the ion velocity along the sensor look direction. The major constituent ion densities are resolved by the mass dependence of the ram energy. The current distribution among the segmented collectors provides the arrival angle of the plasma with respect to the plasma look direction, thus permitting a derivation of the full plasma flow vector ${ }^{10}$.

\section{Langmuir Probe}

Double-Langmuir probes (DLPs) measure the ambient ion density, ion flux, and electron temperature, and electron density ${ }^{11}$. These probes are not to be confused with "double probes," which is a common term in the space physics community referring to two boom-mounted probes for making

DC-AC electric field measurements. Each probe of the DLP is biased with an AC voltage with respect to the other probe, so that ion or electron loss area problems are mitigated. This means that the entire spacecraft body could be made of insulating material and the DLP would still function nominally. This advantage of DLPs is important for 
use on CubeSats, since the exposed conducting surface area can be somewhat small. For a single Langmuir probe to provide accurate results, the spacecraft conducting surface area needs to be sufficiently large as an ion loss area when the single Langmuir probe is biased to electron saturation so that the probe itself doesn't alter the ambient space plasma potential that is being measured. For atomic oxygen, this ratio of electron loss area at the probe tip to ion loss area at the spacecraft surface is $\sim 200: 1$, and $~ 250: 1$ for $\mathrm{NO}^{+}$. The DLP however, is a floating system and electrically isolated from the spacecraft ground/skin. Ion and electron saturation currents that are drawn to the DLP probe tips are effectively drawn between each probe tip, and the electron saturation current drawn in one probe is limited by the ion saturation current drawn in the other probe. A voltage sweep is required for DLPs, as is a transimpedance (current to voltage) amplifier for measuring the current between the two probes of the DLP.

3. Dual-Frequency Crosslink Radio Occultation Experiment 
Radio tomography of the Earth's ionosphere ${ }^{12}$ has enabled near-global observations of the structure and dynamics of key ionospheric regions and processes. MarsCAT will use its telemetry transceivers to measure electron column density data between the various spacecraft, indicative of kilometer-scale density structure. A 10 s data cadence will provide 1000s of ray-paths per orbit from a variety of geometries including occultations near periapsis. 
The radio science technique of measuring the integrated column electron density (or Total Electron Content (TEC)) is accomplished by having a spacecraft transmit coherently phased pairs of radio frequencies to a receiver. The dispersion of the two frequencies through the Martian ionosphere separates their arrival time at the receiver. The differential delay between the two signals gives an accurate measure of the TEC. At Mars, the experiment will use the telemetry transceivers on all MarsCAT spacecraft, each will transmit and receive simultaneously at 390 and $450 \mathrm{MHz}$, frequencies selected to maximize dispersion and reduce the time synchronization burden on the receiver oscillator and microprocessor while also remaining with the frequency band of the Mars Relay CCSDS protocol. The several spacecraft clocks will be synchronized using the microprocessor to align a pseudorandom code sequence, similar to how low-precision GPS receivers maintain accurate time.

\section{Energetic Particles}

We will include low power hybrid pixel radiation imaging detectors from the CERN-based Medipix2

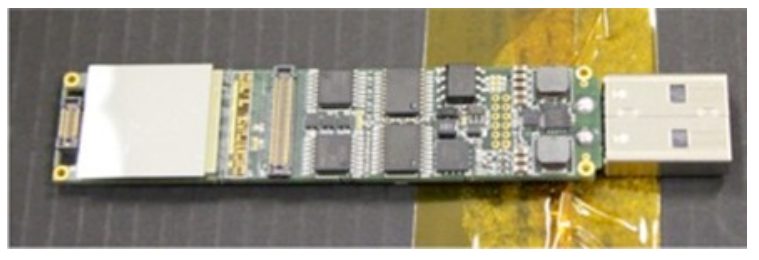

Figure 8. ISS Medipix2 (Timepix) dosimeter Collaboration ${ }^{13}$ on MarsCAT. These detectors can measure energy and arrival directions of electrons and ions from $3 \mathrm{keV}$ to $\mathrm{GeV}$ energies.

\section{Conclusion}

\footnotetext{
${ }^{1}$ Haider, S. A., K. K. Mahajan, and E. Kallio, Mars ionosphere: A review of experimental results and modeling studies, Rev. Geophys., 49, RG4001, doi:10.1029/2011RG000357, 2011.

${ }^{2}$ Jakosky, B. M., R. P. Lin, J. M. Grebowsky, J. G. Luhmann, D. F. Mitchell, G. Beutelschies, T. Priser, M.
} Acuna, L. Andersson, D. Baird, et al. (2015), The Mars Atmosphere and Volatile Evolution (MAVEN) Mission, Space Science Reviews, pp. 1--46.

${ }^{3}$ Bougher, S., et al. (2015), Early MAVEN deep dip campaign reveals thermosphere and ionosphere variability, Science, 350(6261), 0459, doi:10.1126/science.aad0459.

${ }^{4}$ Mitchell, D. L., R. P. Lin, C. Mazelle, H. Rème, P. A. Cloutier, J. E. P. Connerney, M. H. Acuña, and N. F. Ness, Probing Mars' crustal magnetic field and ionosphere with the MGS Electron Reflectometer, J. Geophys. Res., 106(E10), 23419-23427, doi:10.1029/2000JE001435, 2001.

${ }^{5}$ Nielsen E., M. Fraenz, H Zou, J.-S. Wang, D.A. Gurnett, D.L. Kirchner, D.D. Morgan, R. Huff, A. Safaeinili, J.J. Plaut, G Picardi, J.D.Winningham, R.A. Frahm, R. Lundin (2007a), Local plasma processes and enhanced electron densities in the lower ionosphere in magnetic cusp regions on Mars, Planetary and Space Science, 55, 21642172

${ }^{6}$ Fowler C. M., L. Andersson, S. Shaver, R. Lillis, M.E. Usanova, R.E. Ergun, J. McFadden, P.R. Mahay, J.E.P. Connerney, J. Espley, M. Benna, M. Elrod, D.L. Mitchell, C. Mazelle, B.M. Jakosky (2016), Observations of the Rayleigh-Taylor instability in Mars' ionosphere, Submitted to GRL.

${ }^{7}$ Fowler, C. M., L. Andersson, R. E. Ergun, M. Morooka, G. Delory, D. J. Andrews, R. J. Lillis, T. McEnulty, T. D. Weber, T. M. Chamandy, et al. (2015), The first in situ electron temperature and density measurements of the Martian nightside ionosphere, Geophys. Res. Lett., 42, 8854-8861, doi:10.1002/2015GL065267.

${ }^{8}$ Lilensten, J. Bernard, D., Barthélemy, M., Gronoff, G., Wedlund, C. Simon and Opitz, A., "Prediction of blue, red and green aurorae at Mars,” Planetary and Space Science, May 2015, PII : S0032-0633(15)00130-0, DOI : 10.1016/j.pss.2015.04.015

${ }^{9}$ Sheinker, A. and M. B. Moldwin, Adaptive Interference Cancelation using a Pair of Magnetometers, submitted to IEEE Transactions on Aerospace and Electronic Systems, 2015.

${ }^{10}$ Heelis, R. A. and Hanson, W. B. (1998) Measurements of Thermal Ion Drift Velocity and Temperature Using Planar Sensors, in Measurement Techniques in Space Plasmas:Particles (eds R. F. Pfaff, Joseph.E. Borovsky and D. T. Young), American Geophysical Union, Washington, D. C.. doi: 10.1029/GM102p0061

${ }^{11}$ Hershkowitz, Noah. "How Langmuir probes work." Plasma Diagnostics 1 edited by O Auicello and D L Flamm, Academic Press, New York pp113-183, 1989.

${ }^{12}$ Yizengaw, Endawoke, M. B. Moldwin, Peter Lawrence Dyson, and E. A. Essex. "Using tomography of GPS TEC to routinely determine ionospheric average electron density profiles." Journal of Atmospheric and Solar-Terrestrial Physics 69, no. 3 (2007): 314-321.

${ }^{13}$ Kroupa, M., A. Bahadori, T. Campbell-Ricketts, A. Empl, S. M. Hoang, J. Idarraga-Munoz, R. Rios, E. Semones, N. Stoffle, L. Tlustos, D. Turecek and L. Pinsky (2015), A semiconductor radiation imaging pixel detector for space radiation dosimetry, Life Sciences in Space Research, 6, 69-78. 
The MarsCAT Mission is a proposed CubeSat mission to Mars, consisting of several 6U CubeSats equipped with on-board electric propulsion. The mission science goals are aimed at increasing understanding of the ionosphere of Mars, its structure and its dynamics. The mission engineering goals are to demonstrate that it is possible to operate CubeSats in Mars orbit.

\section{References}

